\title{
Inheritance and Protection of Linyi Xiaoguo Clay Sculpture from the Perspective of Harmonious Culture
}

\author{
Pengcheng Shao
}

Feixian College Linyi University, Linyi, Shandong, China

Keywords: Harmonious culture, Xiaoguo clay sculpture, inheritance, protection

\begin{abstract}
Xiaoguo clay sculpture itself is a harmonious integration of the aesthetics and practicality. Regarding to the inheritance and protection, the first is to establish the concept of the harmonious coexistence of artistic value and economic value; and the second is to implement the policy and strategy of combining the traditional craftsmanship with contemporary technology; thus to reach dialectical unity and harmonious perfection inheritance protection, and innovation promoting, so as to form the "harmonious type" cultural heritage brand with core competitiveness.
\end{abstract}

\section{Introduction}

"Harmonious culture" is a cultural system, culture is its way of expression, and harmony is its ideological connotation. It integrates ideals, beliefs, thoughts, norms of behavior, social customs and value orientation, including the overall evaluation and understanding of a harmonious society.

In December 2006, the first batch of non-cultural heritage directories in Shandong Province was announced, and Xiaoguo clay sculpture was in the directories, which attracted the highly attention of the society. However, what end is the status of the development of Xiaoguo clay sculpture? What are the problems? How to further protect, inherit and develop it? This article will study and analyze its protection and inheritance strategies from the perspective of harmonious culture.

\section{Beautiful and Practical Harmony-----Introduction of Xiaoguo Clay Sculpture}

\subsection{Origin}

Xiaoguo clay sculpture is produced in Xiaoguo Village, Xiangcheng Town, Lanyi County, Linyi City, it is named after the origin and it has a history of nearly two hundred years. During the Xianfeng period of the Qing Dynasty, its founder, Mr. Li Zongbiao, first was the disciple of "Clayman Zhang" in Tianjin, and then absorbed the essence of clay sculpture in Huishan Wuxi, and developed the studies with local characteristics by combing with Xiao Guocun's natural conditions and local culture, thus to form her own genre to be circulated till now.

Li Zongbiao's works were not found to be preserved, but he passed down the noble skills of the clay figurines. His son Li Zhanchun and grandson Li Xianzhi, Great-grandson Li Yufa, plus him, the four generations of straight-grandparents and grandchildren were the typical successors of Xiaoguo clay sculpture. Among the fifth generation descendants, Liu Fuxiang and Yin Fangli were typical representatives.

\subsection{Production}

Xiaoguo clay sculpture's production process is mainly divided into two steps: mold printing and paintings. The completion of a work requires nearly forty procedures, which generally include the following steps:

\subsubsection{Molding}

Molding shape is the most important molding method for Xiaoguo clay sculpture, so the first thing is to make a mold. Mold making is divided into two steps: first to make the template, and then is for molding. The prototype of the mold is a template, which is to use the most primitive method to create a clay doll by molding, carving, and pinching a piece of mud. Molding is to press the mud and print it on the template, and then to print it into a mold to get a complete mold. 


\subsubsection{Mudding}

Mudding is the key section to make the clay sculpture. Fine, uniform, clean, and sticky clay is the best choice. In order to enhance the viscosity and flexibility of the mud, a certain proportion of cotton wool should be added to the mud; and then is to rub, throw and pedal the mud repeatedly until the mud is delicate, even, and not sticky to the feet. Thus the strong and flexible mud is finished, in order to prevent the loss of moisture, and the finished mud should be stored in plastic bags.

\subsubsection{Mold printing}

The so-called mold printing is to press the well processed mud into the processed mold to print it into shape.

\subsubsection{Whistle installation}

The whistle installed on the mud shape is the whistle installation. Xiaoguo clay sculpture is a harmonious body of "shape", "color" and "tone". A hole should be first made on the back of the mud shape for the air circulation inside the mud shape, and it is also to prevent the deformation of the mud shape caused by changes in internal and external air pressure. The whistle is generally installed on the bottom of undried clay.

\subsubsection{Polishing}

Polishing is the final processing of the semi-finished products, which is to beautify and modify the defects of the undried mud shape. After polishing, the mud shape is placed at the shadow position for drying, which is to prepare for coloring.

\subsubsection{Coloring}

Xiaoguo clay sculpture has always been said "seven-point color, three-point shaping." The shape of the mold printing only outlines the contour of things without drawing the details. The vivid drawing of various colors can make up for their deficiencies. Therefore, the key to the success of a clay sculpture is coloring, which is also the most important link in the production process.

\subsection{Beautiful appearance}

\subsubsection{Subject features of Xiaoguo clay sculpture}

Subjects come from a wide range of sources, including mainly the animals, theatrical figures, and historical stories, such as dolls with hairpin, lying on the ice for carp, the God of Wealth, Li Kui, the Monkey King, Kong Ming, chickens, tigers, and dogs, etc.

\subsubsection{Modeling features of Xiaoguo clay sculpture:}

The first is to reflect reality by exaggeration. Such as the toy "girl holding flowers", the head height almost equals to that of the body, and the four big strokes of pink can be used to identify the hands and feet.

The second is elegant in gaudy. In order to reflect the simple and friendly rustic flavor of Xiaoguo clay sculpture, the back side of Xiaoguo clay sculpture intentionally retains the earthy color, which is deeply loved by the labors.

\subsubsection{Color characteristics of Xiaoguo clay sculpture:}

Xiaoguo clay sculpture is vivid in color, and it is concise and beautiful, contrasting vividly and fully highlighting its painting features. When using a variety of colors to "make-up", there are a few muddy colors left on the bottom and back, which is very casual at first glance, but actually originality.

From the point of view of function and use, any work cannot be simply categorized into one, and most of them have two or three functions.

\subsection{Function}

From the point of view of function and use, any work cannot be simply considered as one category, and most of them have two or three functions. It is divided into three types: Entertainment Toys, Ornament Type, Beliefs and Worship Type:: 


\section{Harmony between Artistic Value and Economic Value----Concept}

3.1 Learning from ceramic industry---- not only to produce the daily necessities such as plates and bowls, but also to cultivate master craftsmen to produce high-quality artworks

At the end of September 2010, Mr. Zhang Songmao, a master of contemporary art, made a bid for the painting of "Three Visits to the Hut" (2), which was eventually sold for 13 million yuan. Following the record of 1.5 million yuan in 2005 (one of the "three visits to the Hut" series), the new Jingdezhen ceramic art price record was born, creating the new history of ten million of the contemporary ceramic art.

However, daily items such as plates, bowls, spoons, and saucers cost just a few yuan. In the ceramics industry, it is not only with the industrialized production of daily necessities such as plates, bowls, dishes, and spoons, but also the cultivation of the master craftsmanship to produce high-quality artworks. Why can't the clay sculpture industry do in this way?

\subsection{To achieve economic value as much as possible based on the cultural inheritance}

In order to realize the better inheritance and protection of Xiaoguo clay sculpture, the first is to establish a clear sense of harmony for the interaction between culture and economy, and to develop under the premise of not losing its connotation. The starting point for the protection of cultural heritage is for the protection of the culture, and all economic development and utilization measures must return to this center. However, it's unfeasible for goods without the market, the goods without market is just like stranded ships that cannot sail far away. Simply, such goods can't generate the profits. No one would like to invest energy and time into products without benefits. For folk artists and their representative cultural heritage, it is even more impossible to be passed on from one generation to another, and so it is necessary to realize its economic value as much as possible based on cultural inheritance.

\section{Harmony between Traditional Craftsmanship and Contemporary Technology----Policies and Strategies}

The harmonious integration of traditional craftsmanship and contemporary technology can promote the positive and rapid development of Xiaoguo clay sculpture. Xiaoguo clay sculpture must keep pace with the times, and realize the long-term development with the innovation of technology, thus to always shine brightly.

\subsection{The distinctive features of traditional craftsmanship should be maintained for benign development}

The traditional craftsmanship refers to the traditional color varieties and production techniques of Xiaoguo clay sculpture, which have been introduced in details in the first section and will not be repeated here.

4.2 Contemporary technology like mechanized production, electronic materials, digitalized design, and e-commerce sales.

\subsubsection{Mechanized production}

For example, the modern machinery is used for beating and stirring mud during the production process, which can save labor time, labor intensity and human resources, thereby saving production costs.

\subsubsection{Electronic materials}

For example, the electronic technology is used to change the sound of the single-hole reed whistle to the music of electronic devices, thus to attract contemporary people's pursuit of fashion.

\subsubsection{Digitalized design}

For example: To use CAD software to design the model on the computer.

\subsubsection{E-commerce sales}


For example: To build their own sales site and open flagship stores in Tmall, JD, etc.

\section{Harmony between Inheritance Protection and Innovation Promoting----Prospects}

The harmonious coexistence of inheritance protection and innovation promoting is the basic principle of the intangible cultural heritage protection, which is the foundation of protection and inheritance of Xiaoguo clay sculpture, and an innovation in the protection, management and management of cultural heritage protection. It can fully reflect the uniform of the cultural value and economic value of Xiaoguo clay sculpture; it can maximize the protection and utilization benefits of cultural heritage; and it is of great significance to form a "harmonious" cultural heritage brand with core competitiveness.

\section{Acknowledgement}

This article is one of the staged achievements of 2017 Art Education Special Project in Shandong Province: Inheritance and Protection of Linyi Xiaoguo Clay Sculpture from the Perspective of Positive Culture (YJ1711059).

\section{References}

[1] Wang Meng, Application of Cangshan Xiaoguo Clay Sculpture in Art Teaching in Local Elementary Schools [D], Ludong University, 2015.

[2] Liu Jing, Investigation and Research on the Inheritance Activities of Xiaoguo Clay Sculpture in Cangshan County, Shandong Province [D], Northwest University for Nationalities, 2013.

[3] Zhang Yongmei, Artistic Characteristics and Development Research on Folk Clay Sculpture Toys in Linyi, Shandong Province[J], Times Literature (first half month of February), 2009(03): 140-142.

[4] Liu Hong, Exploration of Xiaoguo Clay Sculpture Art[J], Journal of Linyi Teachers College, 2007(05):137-140. 